\title{
MORPHISMS OF MISLIN GENERA INDUCED BY FINITE NORMAL SUBGROUPS
}

\author{
P. J. HILTON and P. J. WITBOOI
}

Received 20 March 2002

\begin{abstract}
We correct an erroneous statement about induced morphisms of Mislin genera and give the correct statement, even under more general hypotheses.
\end{abstract}

2000 Mathematics Subject Classification: 20F18, 20 E34.

As in [9], we denote the class of all finitely generated groups with finite commutator subgroups by $\mathscr{C}_{0}$, and for an $\mathscr{X}_{0}$-group $H$, we let $\chi(H)$ be the set of isomorphism classes of groups $K$ for which $K \times \mathbb{Z} \cong H \times \mathbb{Z}$. If $H$ is a nilpotent $\mathscr{X}_{0}$-group, the Mislin genus (i.e., the genus as defined in [4]) of $H$ is denoted by $\mathscr{G}(H)$. By a result of Warfield [6], we know that if $H$ is a nilpotent $\mathscr{X}_{0}$-group, then $\chi(H)=\mathscr{G}(H)$. Furthermore, for an $\mathscr{X}_{0}$-group $H$, in [9] it is shown that there is an abelian group structure on $\chi(H)$ which coincides with the Hilton-Mislin group structure [3] on $\mathscr{G}(H)$ if $H$ is nilpotent.

In [8, Section 3], it was shown how to define a function $\eta: \chi(H) \rightarrow \chi(H / F)$ if $H$ is an infinite $\mathscr{X}_{0}$-group and $F$ is a finite normal subgroup of $H$. It was also shown that the function is not always a homomorphism [8, Example 5.4]. This is in conflict with [2, Theorem 1.3]. In fact there is an error in [2, Theorem 1.1] in that the function $\alpha_{*}: \mathscr{G}(N) \rightarrow \mathscr{G}(N / F)$ is not always well defined. The counterexample of [9] suggests a way to show explicitly how things may go wrong. (To merely show that $\alpha_{*}$ is not always well defined there are simpler examples, but for a simpler example one may find that there is nevertheless some epimorphisms $\mathscr{G}(N) \rightarrow \mathscr{G}(N / F)$.) We will show that the results of $[2$, Section 1] remain valid.

In order to ensure that the relation $\alpha_{*}$ of [2, Section 1] is a well-defined function, we could follow the option of replacing the domain $\mathscr{G}(N)$ with a different set, which we briefly describe as follows.

Let $\mathcal{N}_{0}$ be the subclass of $\mathscr{X}_{0}$ consisting of all infinite nilpotent groups. For an $\mathcal{N}_{0}$ group $H$ and a suitable finite group $F$, we fix a monomorphism $h: F \rightarrow H$ with $h(F) \triangleleft H$. Now let $K$ be a group in the Mislin genus of $H$, and let $k: F \rightarrow K$ be any monomorphism with $k(F) \triangleleft K$ which admits, for every prime $p$, an isomorphism $f: K_{p} \rightarrow H_{p}$ for which $f \circ k_{p}=h_{p}$. We denote the class of all such pairs $(K, k)$ by $\mathscr{H}_{0}$. If $l: F \rightarrow L$ is another such homomorphism, then we say that $l \sim k$ if there is an isomorphism $\phi: L \rightarrow K$ for which $\phi \circ l=k$. Then $\sim$ is an equivalence relation. Let $\mathscr{G}(H, h)$ be the $\operatorname{set} \mathscr{G}(H, h)=\mathscr{H}_{0} / \sim$ of all equivalence classes of such endomorphisms. Since $\mathscr{G}(H)$ is finite and since there are only finitely many embeddings of $F$ into $H$, it is easy to prove that $\mathscr{G}(H, h)$ is a finite set. At least then we can follow [2, Theorem 1.1]. The association $(K, k) \mapsto K / k(F)$ determines a function $\alpha_{*}: \mathscr{G}(H, h) \mapsto \mathscr{G}(H / h(F))$. There is of course the difficulty that 
the set $\varphi(H, h)$ is not well understood, for example, we do not know whether $\mathscr{G}(H, h)$ has a suitable group structure. Anyway, we are interested in $\mathscr{G}(H)$, and we will follow a different option.

We know (see, e.g., [7]) that if $F$ is a characteristic subgroup of the torsion subgroup $T_{H}$ of $H$, then we do have a homomorphism $\varphi(H) \rightarrow \mathscr{G}(H / F)$, in fact, an epimorphism. In the calculation that leads up to [2, Theorem 3.1], the subgroup $\operatorname{ker} \alpha$ of $N$ that is being factored out is, indeed, a characteristic subgroup of $T$ (see Proposition 7). Further we note that $\tilde{N}$ is of the form $H \times\left(\mathbb{Z}_{2}\right)$ for some group $H$, and then by [7, Corollary 4.2] we have an isomorphism $\mathscr{G}(H) \rightarrow \mathscr{G}(\tilde{N})$. For such a group $H$ we have (see [1]) that $\mathscr{G}(H)=\left(\mathbb{Z}_{\tilde{t}}\right)^{*} /\{1,-1\}$. Thus it follows that [2, Theorem 3.1] is valid. In this paper, we will find a more general condition on the pair $F \triangleleft H$ in order to have a homomorphism $\mathscr{G}(H) \rightarrow \mathscr{G}(H / F)$, in fact, an epimorphism. Our result in this regard is more general in that we do not require the group $H$ to be nilpotent.

We recall the following invariant of an $\mathscr{X}_{0}$-group.

Definition 1 (see [9]). For an $\mathscr{L}_{0}$-group $H$, let $n_{1}$ be the exponent of the torsion subgroup $T_{H}$, let $n_{2}$ be the exponent of the group $\operatorname{Aut}\left(T_{H}\right)$, and let $n_{3}$ be the exponent of the torsion subgroup of the center of $H$. We define the natural number $n(H)=n_{1} n_{2} n_{3}$.

Note that if $H$ is an $\mathscr{X}_{0}$-group and $K$ is a group for which $K \times \mathbb{Z} \cong H \times \mathbb{Z}$, then $K$ is also an $\mathscr{X}_{0}$-group and $T_{K} \cong T_{H}$, so that $n(K)=n(H)$. Also note that for such groups $H$ and $K$, if $\epsilon: H \rightarrow K$ is an embedding then the index $[K: \epsilon(H)]$ is finite.

THEOREM 2. Let $H$ be an infinite $\mathscr{X}_{0}$-group, and let $n=n(H)$. Let $F$ be a finite subgroup of $H$. The following two conditions are equivalent:

(1) given any embedding $\phi: H \rightarrow H$ such that $[H: \phi(H)]$ is relatively prime to $n$, $\phi(F)=F$;

(2) if $L$ is any group for which $L \times \mathbb{Z} \cong H \times \mathbb{Z}$, and $\beta_{1}$ and $\beta_{2}$ are any two embeddings of $L$ onto subgroups $K_{1}$ and $K_{2}$, respectively, of $H$, with both $\left[H: K_{1}\right]$ and $\left[H: K_{2}\right]$ relatively prime to $n$, then $\beta_{1}^{-1}(F)=\beta_{2}^{-1}(F)$.

Proof. Assume that condition (1) holds and suppose that we are given $L, \beta_{1}$, and $\beta_{2}$ as in (2). Then $F$ is contained in both $K_{1}$ and $K_{2}$. In order to prove (2), it suffices to show that, given any isomorphism $\beta: K_{1} \rightarrow K_{2}, \beta(F)=F$. By [9, Theorem 4.2] it follows that there is an embedding $\gamma: H \rightarrow K_{1}$ such that $\left[K_{1}: \gamma(H)\right]$ is relatively prime to $n$ (note that $n(H)=n\left(K_{1}\right)$ ). Let $\epsilon: K_{1} \rightarrow H$ and $\delta: K_{2} \rightarrow H$ be the inclusions. Then we have embeddings $\epsilon \circ \gamma$ and $\delta \circ \beta \circ \gamma$ of $H$ into $H$. By (1), it follows that $\epsilon \circ \gamma(F)=F$ and $\delta \circ \beta \circ \gamma(F)=F$. Moreover, $\epsilon(F)=F$ and $\delta(F)=F$, and consequently we have $\beta(F)=F$. So we have proved that (1) implies (2).

The converse implication is clear.

REMARK 3. Notice that for any infinite $\mathscr{L}_{0}$-group $H$ and any group $L$ for which $L \times \mathbb{Z} \cong H \times Z, L$ is an $\mathscr{L}_{0}$-group and $n(L)=n(H)$. It is then not hard to see that conditions (1) and (2) of Theorem 2 are equivalent to the following condition:

(3) if $\beta_{1}$ and $\beta_{2}$ are any two embeddings of $H$ onto subgroups $K_{1}$ and $K_{2}$, respectively, of $L$, with $\left[L: K_{1}\right]$ and $\left[L: K_{2}\right]$ relatively prime to $n$, then $\beta_{1}(F)=\beta_{2}(F)$. 
We are now able to state and prove a significant result on induced morphisms.

THEOREM 4. Let $H$ be an $\mathscr{X}_{0}$-group, and let $n=n(H)$. Let $F$ be a finite subgroup of $H$ with the property that, given any embedding $\phi: H \rightarrow H$ such that $[H: \phi(H)]$ is relatively prime to $n, \phi(F)=F$. Then, for subgroups $K$ of $H$ with $[H: K]$ relatively prime to $n$, the association $K \mapsto K / F$ defines an epimorphism $\eta: \chi(H) \rightarrow \chi(H / F)$.

Proof. We first note that, by implication, $F$ must be a normal subgroup of $H$. By the equivalence of (1) and (2) in Theorem 2, it follows that $\eta$ is well defined. The proof is completed in a way similar to the proof of [7, Theorem 2.1] using [9, Proposition 6.1].

For an $\mathscr{L}_{0}$-group $H, T_{H}$ has finite characteristic subgroups $\left[T_{H}, T_{H}\right]$ and $Z T_{H}$ to which [7, Theorem 2.1] applies. We point out some other subgroups to which the more general Theorem 4 is applicable.

THEOREM 5. Let $H$ be an infinite $\mathscr{X}_{0}$-group. Let $F=[H, H] \cap T_{H}$. Then $H$, together with $F$, satisfies condition (1) of Theorem 2.

Proof. Let $\phi: H \rightarrow H$ be any embedding such that $[H: \phi(H)]$ is relatively prime to $n$. Then $\phi[H, H]=[\phi H, \phi H]<[H, H]$. Also $\phi\left(T_{H}\right)<T_{H}$. Thus $\phi(F)<F$. Since $F$ is finite, it follows that $\phi(F)=F$.

THEOREM 6. Let $H$ be an infinite $\mathscr{X}_{0}$-group. Let $F=Z H \cap T_{H}$. Then $H$ together with $F$ satisfies condition (1) of Theorem 2.

Proof. Let $\phi: H \rightarrow H$ be any embedding such that $[H: \phi(H)]$ is relatively prime to $n$. Then $\phi$ can be extended to an isomorphism $\psi: H \times \mathbb{Z}^{k} \rightarrow H \times \mathbb{Z}^{k}$ for some $k \in \mathbb{N}$ (see the proof of [9, Theorem 4.1]). Now $Z\left(H \times \mathbb{Z}^{k}\right)=(Z H) \times \mathbb{Z}^{k}$. Since the isomorphism $\psi$ preserves centers and preserves torsion, it follows that $\psi(F)=F$. Since the induced homomorphism $\phi$ maps $T_{H}$ isomorphically onto $T_{H}$, it follows that $\phi(F)=F$.

The following result offers an alternative approach to [2, Theorem 3.1], or to a generalization of it.

Proposition 7. Let $n \in \mathbb{N}$, and let

$$
T=\left\langle x, y, z \mid x^{2}=y^{2}=z^{2 n}=1,[x, y]=z^{n},[x, z]=1=[y, z]\right\rangle .
$$

Then the subgroup $F=\left\langle x, y, z^{n}\right\rangle$ of $T$ is a characteristic subgroup of $T$.

Proof. We note that $F$ is generated by elements of order 2 and every element of order 2 in $T$ is contained in $F$. Therefore $F$ is a characteristic subgroup of $T$.

Proposition 8. Let $n, u \in \mathbb{N}$ be such that $u$ is relatively prime to $2 n$. Let $t$ be the multiplicative order of $u \bmod 2 n$, and let $\tilde{t}$ be the multiplicative order of $u \bmod n$. Let $T$ and $F$ be the groups of Proposition 7 , and let $\zeta$ be the action of $\mathbb{Z}$ on $T$ defined (for $a \in \mathbb{Z})$ by

$$
(a, z) \longmapsto z^{\left(u^{a}\right)}, \quad(a, x) \longmapsto x, \quad(a, y) \longmapsto y
$$


Then, for the group $H=T \rtimes_{\zeta} \mathbb{Z}, F \triangleleft H$ and we have an epimorphism $\chi(H) \rightarrow \chi(H / F)=$ $\left(\mathbb{Z}_{\tilde{t}}\right)^{*} /\{1,-1\}$.

In particular, if $\tilde{t}=t$, then $\chi(H) \simeq \chi(H / F)$.

Proof. Our conditions ensure that indeed $\zeta$ is an action. By Proposition $7, F$ is a characteristic subgroup of $T$, and thus by Theorem 4 , there is an epimorphism $\chi(H) \rightarrow$ $\chi(H / F)$. The group $H / F$ is isomorphic to the group

$$
\left\langle a, b \mid a^{n}=1, b a b^{-1}=a^{u}\right\rangle
$$

and therefore by [5, Theorem 3.8] we have $\chi(H / F)=\left(\mathbb{Z}_{\tilde{t}}\right)^{*} /\{1,-1\}$.

By [8, Theorem 2.6] there is an epimorphism

$$
\left(\mathbb{Z}_{t}\right)^{*} /\{1,-1\} \rightarrow \chi(H)
$$

and so, if $\tilde{t}=t$, then $\chi(H) \simeq \chi(H / F)$.

\section{REFERENCES}

[1] C. Casacuberta and P. Hilton, Calculating the Mislin genus for a certain family of nilpotent groups, Comm. Algebra 19 (1991), no. 7, 2051-2069.

[2] P. Hilton, On induced morphisms of Mislin genera, Publ. Mat. 38 (1994), no. 2, 299-314.

[3] P. Hilton and G. Mislin, On the genus of a nilpotent group with finite commutator subgroup, Math. Z. 146 (1976), no. 3, 201-211.

[4] G. Mislin, Nilpotent groups with finite commutator subgroups, Localization in Group Theory and Homotopy Theory, and Related Topics (Sympos., Battelle Seattle Res. Center, Seattle, Wash., 1974), Lecture Notes in Math., vol. 418, Springer, Berlin, 1974, pp. 103-120.

[5] D. Scevenels and P. Witbooi, Non-cancellation and Mislin genus of certain groups and $\mathrm{H}_{0}$ spaces, J. Pure Appl. Algebra 170 (2002), no. 2-3, 309-320.

[6] R. B. Warfield Jr., Genus and cancellation for groups with finite commutator subgroup, J. Pure Appl. Algebra 6 (1975), no. 2, 125-132.

[7] P. J. Witbooi, Epimorphisms of non-cancellation groups, in preparation.

[8] _ Non-cancellation for groups with non-abelian torsion, in preparation.

[9]___ Generalizing the Hilton-Mislin genus group, J. Algebra 239 (2001), no. 1, 327-339.

P. J. HiLton: SUNY AT BINGHAMTON, BINGHAMTON, NY 13902-6000, USA

Current address: UNIVERSITY OF CENTRAL FloRIDA, ORLANDO, FlORIDA 32816, USA

E-mail address: marge@math. binghamton .edu

P. J. Witbooi: University of the Western CaPe, Private Bag X17, 7535 Bellville, South AFRICA

E-mail address: pwi tbooi@uwc.ac.za 


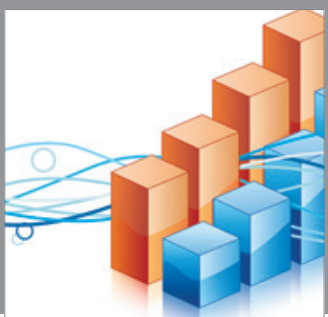

Advances in

Operations Research

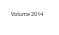

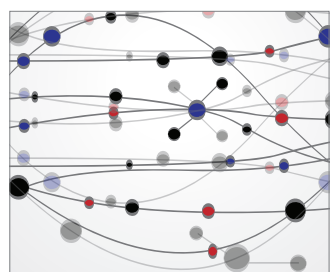

\section{The Scientific} World Journal
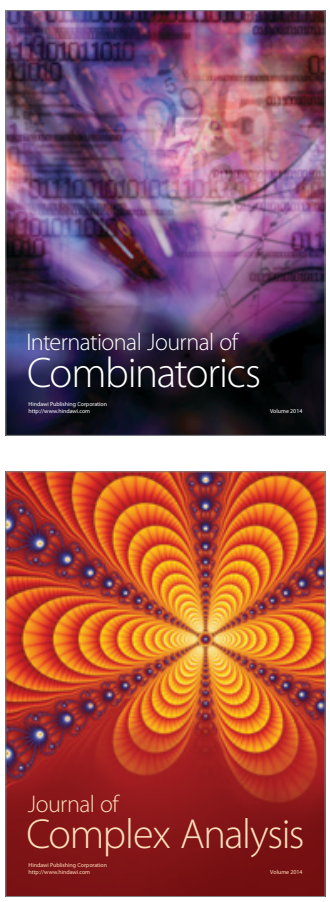

International Journal of

Mathematics and

Mathematical

Sciences
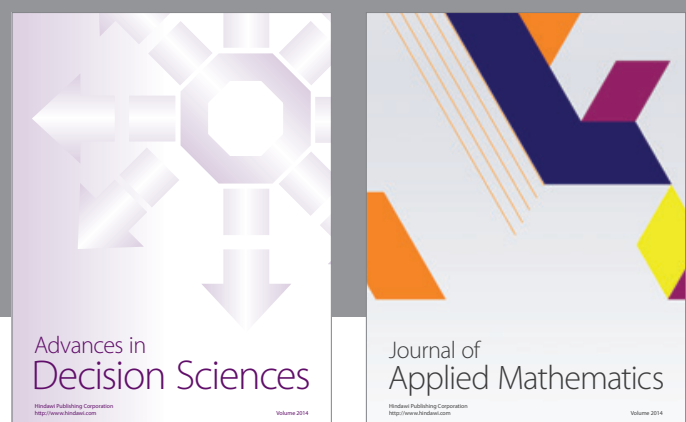

Journal of

Applied Mathematics
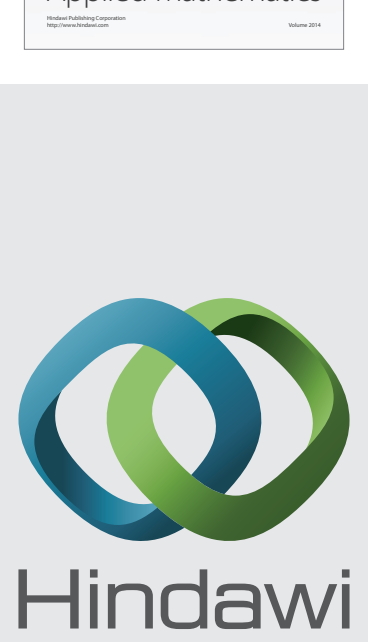

Submit your manuscripts at http://www.hindawi.com
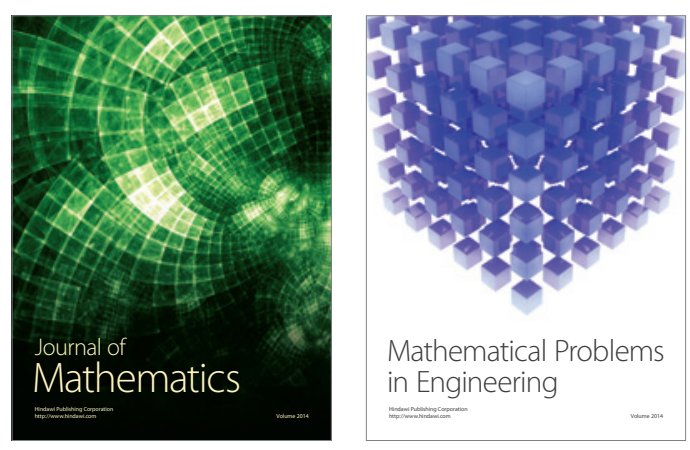

Mathematical Problems in Engineering
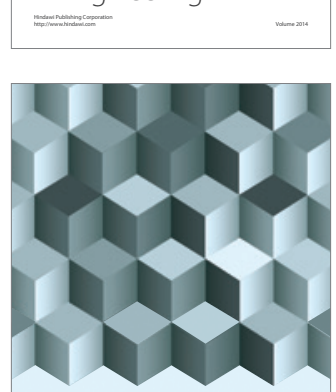

Journal of

Function Spaces
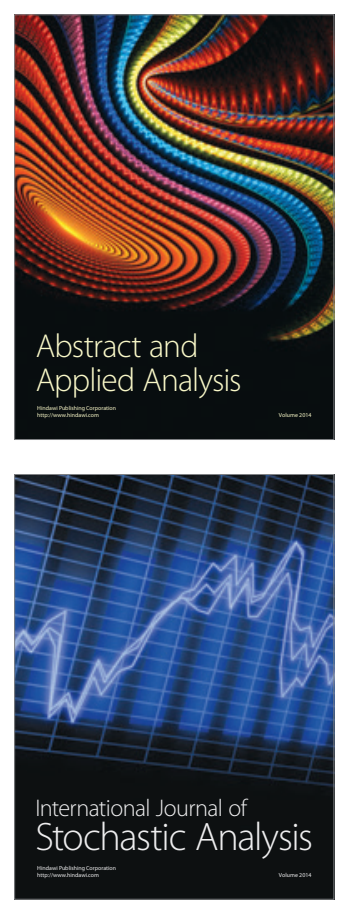

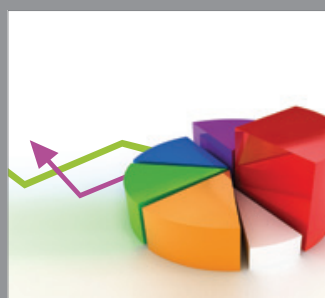

ournal of

Probability and Statistics

Promensencen
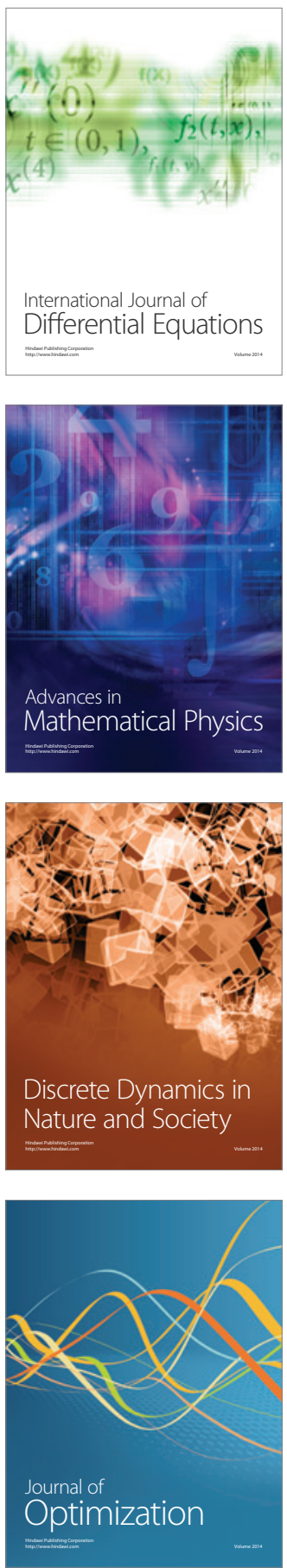